\title{
VECTOR VALUED MULTIPLIERS AND APPLICATIONS
}

\author{
BY N. M. RIVIÈRE ${ }^{1}$
}

Communicated by Gian-Carlo Rota, May 6, 1968

Let $x=\left(x_{1}, \cdots, x_{n}\right) \in R^{n} ; \xi=\left(\xi_{1}, \cdots, \xi_{m}\right) \in R^{m}$. We define $\mathbb{Z}^{p} X^{2}=\left\{f ; f: R^{n+m} \rightarrow C\right.$, such that

$$
\left.\Xi^{p} X^{2}(f)=\left\{\int_{R^{m}}\left[\int_{R^{n}}|f(x, \xi)|^{2} d x\right]^{p / 2} d \xi\right\}^{1 / p}<\infty\right\} .
$$

We shall call $C_{0}^{\infty}\left(R^{n+m}\right)$ the class of infinitely differentiable functions in $R^{n+m}$ with compact support. For $f \in \Xi^{1} X^{1}$ define the Fourier transform of $f$ by

$$
F(f)(y, \eta)=\int_{R^{n+m}} \exp (2 \pi i(x \circ y+\xi \circ \eta)) f(x, \xi) d x d \xi,
$$

where $x \circ y=\sum_{j=1}^{n} x_{j} y_{j}$.

Similarly we define the anti-Fourier transform

$$
\mathfrak{F}^{-1}(f)(y, \eta)=\int_{R^{n+m}} \exp (-2 \pi i(x \circ y+\xi \circ \eta)) f(x, \xi) d x d \xi .
$$

We shall denote by $\chi_{E}(x, \xi)$ the characteristic function of the set $E$. Finally for $f \in C_{0}^{\infty}\left(R^{n+m}\right)$ and $g(x, \xi)$ bounded we define

$$
T(f)=\mathfrak{F}^{-1}(g \mathfrak{F}(f)) .
$$

Theorem 1 (Littlewood-Paley). Let $\Lambda=\left(\lambda_{1}(x), \cdots, \lambda_{m}(x)\right.$ ) denote an m-vector of real valued functions. For the multi-index $N=\left(n_{1}, \cdots, n_{m}\right)\left(n_{\mathrm{s}}= \pm 1, \pm 2, \cdots\right)$ define

$$
Q_{N}=\left\{(x, \xi) ; 2^{n_{s}} \leqq\left|\xi_{s}-\lambda_{s}(x)\right| \leqq 2^{n_{s}+1} ; 1 \leqq s \leqq m\right\} .
$$

Consider $f \in \mathbb{\Xi}^{p} X^{2}$, and set $f_{N}=\mathfrak{F}^{-1}\left(X_{Q_{N}} \mathfrak{F}(f)\right)$. Then

$$
\begin{aligned}
& B_{p}^{-m} \Xi^{p} X^{2}\left(\left\{\sum_{N}\left|f_{N}\right|^{2}\right\}^{1 / 2}\right) \leqq \Xi^{p} X^{2}(f) \\
& \quad \leqq B_{p}^{m} \Xi^{p} X^{2}\left(\left\{\sum_{N}\left|f_{N}\right|^{2}\right\}^{1 / 2}\right), \text { for all } p, 1<p<\infty
\end{aligned}
$$

( $B_{p}$ depends on p only).

${ }^{1}$ Research partially supported by NSF GP 7041X. 
TheOREM 2. Let $Q_{N}$ be as in Theorem 1 and assume $g(x, \xi)$ is a bounded measurable function such that

$$
\frac{\partial^{m}}{\partial \xi_{1} \cdots \partial \xi_{m}}\left(x Q_{N} g\right)=\mu_{N}(x, \xi) \quad \text { is a finite measure. }
$$

(The last equality is to be understood in the sense of distributions.) Then for $f \in C_{0}^{\infty}\left(R^{n+m}\right)$ and for $T(f)=\mathscr{F}^{-1}(g F(g))$ we have

$$
\Xi^{p} X^{2}(T f) \leqq B_{p}^{m}\left[\sup _{N}\left\{\sup _{x \in R_{n}} \int_{R_{m}} d\left|\mu_{N}(x, \xi)\right|\right\}\right] \Xi^{p} X^{2}(f)
$$

for all $p, 1<p<\infty$.

As a consequence of Theorem 2 we obtain:

THEOREM 3. Let $\mathscr{L}=\left\{l_{1}, \cdots, l_{r}\right\}$ be a finite family of affine functionals from $R^{m}$ into $R$, and assume $S \subset R^{n+m}$ has the property that

$$
S \cap\left\{\left(x_{0}, \xi\right), x_{0} f i x e d\right\}=\left\{\xi ; l_{j}(\xi) \geqq \lambda_{j}\left(x_{0}\right) ; 1 \leqq j \leqq r\right\} .
$$

$\operatorname{Set} g(x, \xi)=\chi_{8}(x, \xi)$. Then

$$
\Xi^{p} X^{2}(T f) \leqq B_{p}^{r m} \Xi^{p} X^{2}(f) .
$$

In particular if $m=1$ and $S \subset R^{n+1}$ is a finite union of disjoint convex sets (say $k$ sets) then

$$
\Xi^{p} X^{2}(T f) \leqq B_{p} k \Xi^{p} X^{2}(f) ; \text { for all } p, \quad 1<p<\infty .
$$

REMARK 1. The result of Theorem 3 is the best possible of its kind. More explicitly, if $S=\left\{(x, \xi) ; x \in R^{n}, \xi \in R\right.$; such that $\left.|x|^{2}+\xi^{2} \leqq 1\right\}$ and $T(f)=\mathscr{F}^{-1}\left(\chi_{8} \mathcal{F}(f)\right)$ is a bounded operator from $\Xi^{p} X_{1}^{q_{1}} \cdots X_{n}^{q_{n}}$ into itself for all $p, 1<p<\infty$; then $q_{1}=q_{2}=\cdots=q_{n}=2$. This result is essentially known and due to C. S. Herz [2, p. 996], who shows that $T$ is not a bounded operator from $L^{p}\left(R^{n+1}\right)$ into itself when $p \leqq 2(n+1) /(n+2)$ or $p \geqq 2(n+1) / n$. The proof can be extended to show the above result (see also Theorem 5 ).

Another application of Theorem 2 is the following theorem

Theorem 4. Let $P(x, \xi)$ and $Q(x, \xi)$ be two polynomials in the $\xi-$ variable $\left(x \in R^{n}, \xi \in R\right)$ of degrees $m_{1}$ and $m_{2}$ respectively. Assume that $g(x, \xi)=P(x, \xi) / Q(x, \xi)$ is a bounded measurable function.

$$
\Xi^{p} X^{2}(T f) \leqq B_{p}\left(m_{1}+m_{2}\right) \Xi^{p} X^{2}(f) \text { for all } p, \quad 1<p<\infty .
$$


REMARK 2. As in the case of Theorem 3, the result of Theorem 4 is the best possible of its kind. In [3] W. Littman, C. McCarthy and the author prove that $g(x, \xi)=\left(|x|^{2}-\xi+i\right)^{-1}$ is not a multiplier in $L^{p}\left(R^{n+1}\right)$ for either $p<2(n+1) /(n+2)$ or $p>2(n+1) / n$; once again the main estimate of the proof actually shows that the conclusion of Remark 1 is equally valid here (see also Theorem 5).

Using some basic results of the Riesz theory of interpolation for spaces of mixed norm (see [1]), it is possible to extend the results of Theorems 3 and 4.

Given two Banach spaces $B_{0}$ and $B_{1}$, we shall denote by $\left[B_{0}, B_{1}\right]_{\alpha}(0 \leqq \alpha \leqq 1)$ the $\alpha$-intermediate space of the Riesz interpolation having for end points $B_{0}$ and $B_{1}$.

Set

$$
B_{1}^{(p)}=X_{1}^{p} X_{2}^{2} \cdots X_{n}^{2}
$$

and

$$
B_{j+1}^{(p)}=\left[B_{j}^{(p)}, X_{j+1}^{p} X_{1}^{2} \cdots X_{n}^{2}\right]_{j /(j+1)} .
$$

TheOREM 5. Let $g(x, \xi)$ be either the characteristic function of a finite union of convex sets (as in Theorem 3) ( $x \in R^{n}, \xi \in R$ ) or the bounded ratio of two polynomials in all variables (as in Theorem 4). Then

$$
\|T(f)\|_{B_{n+1}^{(p)}} \leqq B_{p}\|f\|_{L_{q}\left(R^{n+1}\right)} \text { for } 1<p \leqq 2,
$$

where $(n+1) / q=1 / p+n / 2(2 n /(n+2)<q \leqq 2)$.

$$
\|T(f)\|_{L_{q}\left(R^{n+1}\right)} \leqq B_{p}\|f\|_{B_{n+1}^{(p)}} \text { for } 2 \leqq p<\infty,
$$

and $q$ as before $(2 \leqq q<2(n+1) / n)$.

The constant $B_{p}$ depends on $p$ and $T$ as in Theorems 3 and 4 .

The proofs of these results will appear elsewhere.

\section{REFERENCES}

1. A. P. Calderon, Intermediate spaces and interpolation, the complex method, Studia Math. 24 (1964), 113-190.

2. C. S. Herz, On the mean inversion of Fourier and Hankel transforms, Proc. Nat. Acad. Sci. 40 (1954), 996-999.

3. W. Littman, C. McCarthy, N. M. Rivière, The non-existence of $L^{p_{-} \text {-estimates for }}$ certain translation-invariant operators, Studia Math. 30 (1968).

University of MinNesota 